\title{
SOME PROPERTIES OF MATROIDS OBTAINED FROM CONCEPT LATTICE APPROACHES
}

\author{
Hua MaO \\ Department of Mathematics, Hebei University \\ Baoding 071002, China \\ yushengmao@263.net
}

\begin{abstract}
Using the Hasse diagrams of concept lattices, we investigate the relations between matroids and geometric contexts, followed by judging a mathematical construction to be a matroid. We provide an idea to find out the dual of a matroid from the ways of concept lattice drawing. In addition, we utilize the Hasse diagrams of concept lattices to discuss the minors of matroids, direct sum of matroids and the connectivity of a matroid. All the consequences demonstrate that the theory of concept lattice drawing can be used into matroids. This generalizes the applied fields of concept lattices.

Key words and Phrases: Matroid, concept lattice, Hasse diagram, geometric lattice.
\end{abstract}

\begin{abstract}
Abstrak. Pada paper ini kami meneliti relasi antara konteks matroid dan geometrik dengan menggunakan diagram Hasse dari konsep lattice, dilanjutkan dengan pertimbangan suatu konstruksi matematika untuk menjadi suatu matroid. Kami menyajikan suatu ide untuk mendapatkan dual dari suatu matroid dengan cara penggambaran konsep lattice. Sebagai tambahan, kami menggunakan diagram Hasse dari konsep lattice untuk membahas minor dari matroid, jumlah langsung dari matroid dan keterhubungan dari suatu matroid. Semua konsekuensi memperlihatkan bahwa teori penggambaran konsep lattice dapat digunakan pada matroid. Hal ini memperumum bidang-bidang terapan dari konsep lattice.
\end{abstract}

Kata kunci: Matroid, konsep lattice, diagram Hasse, lattice geometrik.

\section{Introduction and Preliminaries}

We know from $[8,14,16]$ that as a branch of combinatorics, a matroid is a structure that captures the generalizations of linear independence in vector spaces.

2000 Mathematics Subject Classification: 05B35; 68R05; 06B75.

Received: 13-07-2016, revised: 10-01-2017, accepted: 10-01-2017. 
There are many equivalent ways to define a matroid, and many notions within matroid theory have a variety of equivalent formulations. Depending on the sophistication of the notion, it may be nontrivial to show that the different formulations are equivalent, a phenomenon sometimes called cryptomorphism significant definitions of a matroid include those in terms of independent sets, circuits, closed sets and hyperplanes.

Some authors have introduced many different algorithms to determine the construction of a matroid such as greedy algorithm (see $[1,8,14,16]$ ). They also provide many algorithms to consider some properties of a matroid such as intersections of matroids and so on (see $[8,14,16]$ ). All of these algorithms bring many results about the structures of matroids and make matroids apply in other fields such as graph theory and optimization theory (see $[1,8,14,16]$ ). Most of these algorithms are relative to independent sets and seldom to the family of closed sets, though the family of closed sets of a matroid has important contents. We find that there are equivalent axioms for matroids in terms of independent sets and closure sets (see $[8,14,16])$. Hence, if we use the closure sets of a matroid, then we may search out new algorithms for matroids. Furthermore, these new algorithms can explore the properties and structures of matroids which have failed to find out before. We may infer the failure reason to only consider with "matroids". If we apply with another theory, then we may success.

Concept lattices have become a useful tool in data analysis and knowledge processing (cf. $[3,4,7,9,10,13,15])$ and can be graphically represented by Hasse diagrams which have been proved as useful communication tools in many applications (cf. $[3,4,13,15])$.

It is well known that matroid have their geometric representation, that is, up to the isomorphism, a simple matroid corresponds to a geometric lattice, and vice versa (see $[8,14,16])$. In addition, every matroid can be simplified. It seems more natural to investigate the relationship between matroid geometric representations and drawing concept lattices. In reality, some researches have utilized concept lattices into matroids with matroid geometric representation (cf. [11,12]). Additionally, we notice that up to the isomorphism, every lattice is determined by its Hasse diagram. Hence, if we explore an approach to deal with matroids with the Hasse diagrams of concept lattices, using the assistance of matroid geometric representations (that is, using the assistance of the families of closed sets of matroids), then we may infer that some constructions and algorithms relative to matroids will be produced. Meanwhile, concept lattices can search out new applied fields.

Actually, we find that isomorphism is useful to consider mathematical structures. In fact, many researchers like to express their ideas up to isomorphism.

This paper presents five applications of ready-made algorithms of drawing concept lattices. First, we explore the relationships between concept lattices and matroids under the isomorphism. Using the relationships and ready-made algorithms for drawing concept lattices, we search out the methods to determining a 
construction to be a matroid. Following these methods, we provide an approach to find out the family of hyperplanes of a matroid. Applying this approach, we present a way to seek out the family of circuits of a matroid and the dual of a matroid. Utilizing the above methods, we explore the constructions of minors of a matroid. Afterwards, we discuss the direct sum of matroids and the connectivity of a matroid. All these discussion are assisted by the drawing of concept lattices, and discussed under the isomorphism.

First of all, we recall some knowledge what we need in the sequel. For more details, please, refer to $[5,6]$ for lattice theory; $[8,14,16]$ for matroid theory; [4] for concept lattices. We assume that throughout this paper, all sets under consideration are finite. For two mathematical structures $\mathfrak{L}_{1}$ and $\mathfrak{L}_{2}, \mathfrak{L}_{1} \cong \mathfrak{L}_{2}$ means that they are isomorphic.

Subsection 1.1 introduces some notations and properties relative to lattices and matroids. We review the notions and lemmas of concept lattices in Subsection 1.2. For convenient, we give a definition in Subsection 1.3.

\subsection{Lattice and Matroid.}

Definition 1.1. [16, p.51 or 15] A finite lattice is geometric if it is semimodular and every point is the join of atoms.

Lemma 1.2. (1) $[16$, p.50] Let $M=(S, \mathcal{F})$ be a matroid with $\mathcal{F}$ as its family of closed sets. Then $(\mathcal{F}, \subseteq)$ is a lattice with $A \wedge B=A \cap B$ and $A \vee B=\cap\{X: X \in$ $\mathcal{F}, A \cup B \subseteq X\}$.

(2) $[16, p .54$ or 8$]$ The correspondence between a geometric lattice $L$ and the matroid $M(L)$ on the set of atoms of $L$ is a bijection between the set of finite geometric lattices and the set of simple matroids.

If $M=(S, \mathcal{F})$ is a matroid, then we sometimes denote $\mathcal{F}$ as $\mathcal{F}_{M}$.

We find from [16, p.54] that for a matroid $M$, there is one and only one simple matroid $M^{\prime}=\left(S, \mathcal{F}^{\prime}\right)$ determined by $M=(S, \mathcal{F})$, and besides, $(\mathcal{F}, \subseteq)$ and $\left(\mathcal{F}^{\prime}, \subseteq\right)$ satisfy $(\mathcal{F}, \subseteq) \cong\left(\mathcal{F}^{\prime}, \subseteq\right)$. Therefore, in what follows, a matroid means a simple matroid.

\subsection{Concept Lattice Theory.}

Definition 1.3. [4, p.19] If $\left(A_{1}, B_{1}\right)$ and $\left(A_{2}, B_{2}\right)$ are concepts of a context $(O, P, I)$, and $A_{1} \subseteq A_{2}$ (which is equivalent to $B_{2} \subseteq B_{1}$ ), we write $\left(A_{1}, B_{1}\right) \leq\left(A_{2}, B_{2}\right)$. The set of all concepts of $(O, P, I)$ ordered in this way is denoted by $\mathcal{B}(O, P, I)$ and is called the concept lattice of the context $(O, P, I)$. 
Lemma 1.4. [4, p.20] The concept lattice $\mathcal{B}(O, P, I)$ is a complete lattice. A complete lattice $V$ is isomorphic to $\mathcal{B}(O, P, I)$ if and only if there are mappings $\gamma: O \rightarrow V$ and $\mu: P \rightarrow V$ such that $\gamma(O)$ is supremum-dense in $V, \mu(P)$ is infimum-dense in $V$ and $g I m$ is equivalent to $\gamma g \leq \mu m$ for all $g \in O$ and all $m \in P$. In particular, $V \cong \mathcal{B}(V, V, \leq)$.

\subsection{New Notions.}

Definition 1.5. A context $(O, P, I)$ is geometric if $\mathcal{B}(O, P, I)$ is geometric.

\section{Applications}

In this section, we will use the Hasse diagrams of concept lattices especially relative to geometric contexts to discuss matroids.

2.1. Relations. There are many ready-made algorithms to draw concept lattices (see $[2,3,4,6,13,15])$. If we analyze the relations between matroids and concept lattices clearly, then we will not need to produce new algorithms for matroids to deal with properties of matroids only using the existed algorithms of drawing concept lattices. These points are the guild-line for the following research.

Lemma 2.1. Let $\mathcal{F}$ be a collection of subsets of a set $S$ and $A \cap B \in \mathcal{F}$ for any $A, B \in \mathcal{F}$. Then $(\mathcal{F}, \subseteq)$ is a lattice with $A \wedge B=A \cap B$ and $A \vee B=\cap\{X: X \in$ $\mathcal{F}, A \cup B \subseteq X\}$.

PROOF. Routine verification.

Theorem 2.2. Let $\mathcal{F}$ be a collection of subsets of a set $S$ and $A \cap B \in \mathcal{F}$ for any $A, B \in \mathcal{F}$. Then under the isomorphism, $(S, \mathcal{F})$ is a matroid if and only if the context $((\mathcal{F}, \subseteq),(\mathcal{F}, \subseteq), \subseteq)($ simply by $(\mathcal{F}, \mathcal{F}, \subseteq))$ is geometric, where $(\mathcal{F}, \subseteq)$ is defined as Lemma 2.1.

Proof. $(\Leftarrow)$ In light of Lemma 1.4 and Lemma 2.1, we obtain $(\mathcal{F}, \subseteq) \cong \mathcal{B}(\mathcal{F}, \mathcal{F}, \subseteq)$. Thus, $(\mathcal{F}, \subseteq)$ is geometric because Definition 1.5 and the geometric of $(\mathcal{F}, \mathcal{F}, \subseteq)$. By virtue of Lemma 1.2(2), we receive that up to the isomorphism, there is a matroid $M=\left(S, \mathcal{F}_{M}\right)$ with $\mathcal{F}_{M}$ as its family of closed sets such that the lattice $\left(\mathcal{F}_{M}, \subseteq\right)$ is isomorphic to $(\mathcal{F}, \subseteq)$, where $\left(\mathcal{F}_{M}, \subseteq\right)$ is defined in Lemma 1.2(1). Furthermore, up to the isomorphism, $(S, \mathcal{F})$ is a matroid.

$(\Rightarrow)$ We can indicate from Lemma 1.2(2) that, up to the isomorphism, $M=$ $(S, \mathcal{F})$ corresponds to a geometric lattice $L(M)$ satisfying $L(M) \cong(\mathcal{F}, \subseteq)$. The lattice $(\mathcal{F}, \subseteq)$ is defined in Lemma 1.2(1). After comparing Lemma 1.2(1) with Lemma 2.1, we find that the lattice $(\mathcal{F}, \subseteq)$ is the same to that in Lemma 2.1. By Lemma 2, we obtain $(\mathcal{F}, \subseteq) \cong \mathcal{B}(\mathcal{F}, \mathcal{F}, \subseteq)$. So, $L(M) \cong \mathcal{B}(\mathcal{F}, \mathcal{F}, \subseteq)$ holds. Hence $(\mathcal{F}, \mathcal{F}, \subseteq)$ is geometric. 
Theorem 2.2 provides a method to determine a construction whether a matroid. Actually, for a collection $\mathcal{F}$ of subsets of a set $S$, we can state the following expressions.

(I) If for some $A, B \in \mathcal{F}$, there is $A \cap B \notin \mathcal{F}$. Then by Lemma $1.2(1),(S, \mathcal{F})$ is not a matroid.

(II) If $A \cap B \in \mathcal{F}$ for any $A, B \in \mathcal{F}$. Then we will do with the following two steps.

The first step is to draw the Hasse diagram of concept lattice $\mathcal{B}(\mathcal{F}, \mathcal{F}, \subseteq)$ using one of the algorithms for drawing concept lattices in $[2,3,4,6,13,15]$. From the Hasse diagram of $\mathcal{B}(\mathcal{F}, \mathcal{F}, \subseteq)$, we can decide the geometry of $\mathcal{B}(\mathcal{F}, \mathcal{F}, \subseteq)$.

The second step is to find the matroidal construction of $(S, \mathcal{F})$ by Definition 1.5 and Theorem 2.2 if $\mathcal{B}(\mathcal{F}, \mathcal{F}, \subseteq)$ is geometric.

In fact, the expressions (I) and (II) taken together implies that we receive some algorithms to construct matroids from $[2,3,4,6,13,15]$ which provide some algorithms for concept lattices drawing.

Let $M=(S, \mathcal{F})$ be a matroid and $\mathbb{K}_{M}$ be a context. We denote by $M \leftrightarrow \mathbb{K}_{M}$ if $\mathcal{B}\left(\mathbb{K}_{M}\right) \cong(\mathcal{F}, \subseteq)$. By Theorem 2.2, we infer that under the isomorphism, we only need to consider $\mathbb{K}_{M}$ if we consider the properties of $M$. The consequences in the following subsections will demonstrate the correct of this point.

2.2. Hyperplanes. Let $\mathcal{F}$ be the collection of subsets of a set $S$ and $A \cap B \in \mathcal{F}$ for any $A, B \in \mathcal{F}$. By Lemma 2.1 and the algorithms in [13,15], we will obtain the Hasse diagram of $\mathcal{B}(\mathcal{F}, \mathcal{F}, \subseteq)$, and therefore the hyperplanes $\mathcal{H}$ of $\mathcal{B}(\mathcal{F}, \mathcal{F}, \subseteq)$. If $\mathcal{B}(\mathcal{F}, \mathcal{F}, \subseteq)$ is geometric, then under the isomorphism, $\mathcal{H}$ is the set of hyperplanes of the matroid $M=(S, \mathcal{F})$ because of $\mathcal{B}(\mathcal{F}, \mathcal{F}, \subseteq) \cong(\mathcal{F}, \subseteq)$. On the other hand, we guarantee from [16, p.39, Theorem 3] that a matroid $M$ is uniquely determined by the set of hyperplanes of $M$.

The above two hands together shows that up to the isomorphism, we can produce a construction of a matroid from the hyperplanes in the Hasse diagram of a geometric context.

2.3. Dual and circuits. We indicate in light of [16, p.9, Theorem 5] that a matroid is determined by its set of circuits. Next we will find a method to construct the dual of a matroid with the assistence of Hasse diagrams of concept lattices of a geometric context.

Let $M$ be a matroid on $S$ with $\mathcal{H}_{M}$ as its set of hyperplanes. We infer to pledge $\mathcal{C}^{*}=\left\{S \backslash H: H \in \mathcal{H}_{M}\right\}$ to be the set of cocircuits of $M$ by view of [16, p.39, Theorem 2]. Hence, we obtain that $\left(S, \mathcal{C}^{*}\right)$ is the dual $M^{*}$ of $M$.

Combining Subsection 2.2 with the above, it follows that up to the isomorphism, with one of the algorithms for drawing concept lattices in $[2,3,4,6,13,15]$, we receive $M^{*}$ directly, and obtain the set of circuits of $M$ according to $\left(M^{*}\right)^{*}=M$. 
2.4. Minors. In Subsections 2.1, 2.2 and 2.3, we discuss three applications of drawing concept lattices. Next we will find an application of drawing concept lattices in the minors of a matroid $M=(S, \mathcal{F})$.

Considering the definitions of minors in [16, p.65] with [16, p.63, Theorem 2] and Subsection 2.3, we find that if we consider the application of drawing concept lattices in the minors of a matroids, we only need to analyze with the restriction $M \mid T$ of $M$ to $T$ where $T \subseteq S$.

Let $M=(S, \mathcal{F})$ be a matroid and $T \subseteq S$. We may easily obtain $(T, \mathcal{F} \mid T)$ where $\mathcal{F} \mid T=\{A \subseteq T$ : there is $F \in \mathcal{F}$ satisfying $A=F \cap T\}$. Obviously, $A \cap B \in \mathcal{F} \mid T$ holds for any $A, B \in \mathcal{F} \mid T$ because $\mathcal{F}$ is closed for the meet. Hence, by Theorem $2.2, \mathcal{F} \mid T$ is the collection of closed sets of a matroid on $T$ if and only if $(\mathcal{F}|T, \mathcal{F}| T, \subseteq)$ is geometric. This determination can be fulfilled with the method in Subsection 2.1. Moreover, combining with Subsection 2.3, we receive methods to produce minors of a matroid from concept lattices drawing algorithms in $[2,3,4,6,13,15]$.

2.5. Direct sum and connection. The fifth application of drawing concept lattices is about direct sum of matroids and connectivity of a matroid. The definition of direct sum $M_{1} \oplus M_{2}$ of two matroids $M_{1}$ and $M_{2}$ is seen [16, p.72]. The definition of direct sum $\mathbb{K}_{1}+\mathbb{K}_{2}$ of two contexts $\mathbb{K}_{1}$ and $\mathbb{K}_{2}$ is in [4, p.46]. The following Theorem 2.3 is the cornerstone for the fifth application.

Theorem 2.3. (1) Let $M_{1}$ and $M_{2}$ be two matroids on sets $S_{1}$ and $S_{2}$ respectively, $\mathbb{K}_{M_{1}}, \mathbb{K}_{M_{2}}$ be the corresponding contexts, that is, $M_{1} \leftrightarrow \mathbb{K}_{M_{1}}$ and $M_{2} \leftrightarrow \mathbb{K}_{M_{2}}$. If $M_{1} \oplus M_{2}$ is true, then up to the isomorphism, $\mathcal{B}\left(\mathbb{K}_{M_{1}}+\mathbb{K}_{M_{2}}\right)=\mathcal{B}\left(\mathbb{K}_{M_{1} \oplus M_{2}}\right)$ is correct.

(2) If $\mathbb{K}_{1}, \mathbb{K}_{2}$ are geometric. Then up to isomorphism, there are matroids $N_{j}, M_{j}^{\prime},(j=1,2)$ and two contexts $\mathbb{K}_{1}^{\prime}$ and $\mathbb{K}_{2}^{\prime}$ such that $N_{j} \leftrightarrow \mathbb{K}_{j}, N_{j} \cong M_{j}^{\prime}, \mathbb{K}_{j} \cong$ $\mathbb{K}_{j}^{\prime}, M_{j}^{\prime} \leftrightarrow \mathbb{K}_{j}^{\prime},(j=1,2)$, and besides, $M_{1}^{\prime} \oplus M_{2}^{\prime}$ holds.

Proof. (1) Lemma 1.2 and Definition 1.5 imply that both $\mathcal{B}\left(\mathbb{K}_{M_{1}}\right)$ and $\mathcal{B}\left(\mathbb{K}_{M_{2}}\right)$ are geometric, and so $\mathcal{B}\left(\mathbb{K}_{M_{1}}\right) \times \mathcal{B}\left(\mathbb{K}_{M_{2}}\right)$ is geometric according to $[16$, p.73, Theorem 1 \&15]. By virtue of [4, p.40], we obtain $\mathcal{B}\left(\mathbb{K}_{M_{1}}+\mathbb{K}_{M_{2}}\right) \cong \mathcal{B}\left(\mathbb{K}_{M_{1}}\right) \times \mathcal{B}\left(\mathbb{K}_{M_{2}}\right)$. Hence, we receive $\mathcal{B}\left(\mathbb{K}_{M_{1}}+\mathbb{K}_{M_{2}}\right)$ to be geometric.

On the other hand, $S_{1} \cap S_{2}=\emptyset$ holds in light of the truth of $M_{1} \oplus M_{2}$. Additionally, owing to the results in [16, pp.72-73], we find that if $M_{1} \oplus M_{2}$ is true, then the following $(\alpha)$ and $(\beta)$ are correct.

( $\alpha) A \in \mathcal{F}_{M_{1} \oplus M_{2}}$ if and only if $A \cap S_{1} \in \mathcal{F}_{M_{1}}$ and $A \cap S_{2} \in \mathcal{F}_{M_{2}}$, where $\mathcal{F}_{M_{1} \oplus M_{2}}, \mathcal{F}_{M_{1}}, \mathcal{F}_{M_{2}}$ is the family of closed sets of $M_{1} \oplus M_{2}, M_{1}, M_{2}$ respectively.

$(\beta)\left(\mathcal{F}_{M_{1}}, \subseteq\right) \times\left(\mathcal{F}_{M_{2}}, \subseteq\right)$ is $\left(\mathcal{F}_{M_{1} \oplus M_{2}}, \subseteq\right)$ up to the isomorphism.

Therefore, from Lemma 1.2, Lemma 1.4 and Theorem 2.2 , we obtain $\mathcal{B}\left(\mathbb{K}_{M_{j}}\right) \cong$ $\left(\mathcal{F}_{M_{j}}, \subseteq\right),(j=1,2)$ and $\mathcal{B}\left(\mathbb{K}_{M_{1} \oplus M_{2}}\right) \cong\left(\mathcal{F}_{M_{1} \oplus M_{2}}, \subseteq\right)$. Hence, we receive $\left(\mathcal{F}_{M_{1}}, \subseteq\right.$ 
)$\times\left(\mathcal{F}_{M_{2}}, \subseteq\right) \cong \mathcal{B}\left(\mathbb{K}_{M_{1}}\right) \times \mathcal{B}\left(\mathbb{K}_{M_{2}}\right)$. Moreover, $\mathcal{B}\left(\mathbb{K}_{M_{1}}\right) \times \mathcal{B}\left(\mathbb{K}_{M_{2}}\right) \cong \mathcal{B}\left(\mathbb{K}_{M_{1} \oplus M_{2}}\right)$ holds.

Summing up the above, we obtain $\mathcal{B}\left(\mathbb{K}_{M_{1}}+\mathbb{K}_{M_{2}}\right)=\mathcal{B}\left(\mathbb{K}_{M_{1} \oplus M_{2}}\right)$ up to the isomorphism.

(2) Since $\mathbb{K}_{j}$ is geometric $(j=1,2)$. By Lemma 1.2 and Theorem 2.2 , up to the isomorphism, there are two matroids $N_{1}$ and $N_{2}$ satisfying $N_{j} \leftrightarrow \mathbb{K}_{j},(j=1,2)$. In fact, $N_{j}$ is produced from $\mathcal{B}\left(\mathbb{K}_{j}\right)$ by the method in [16, p.51, Theorem 1$],(j=$ $1,2)$.

For $\mathbb{K}_{j}=\left(O_{j}, P_{j}, I_{j}\right),(j=1,2)$, we may easily present two contexts $\mathbb{K}_{j}^{\prime}=$ $\left(O_{j}^{\prime}, P_{j}^{\prime}, I_{j}^{\prime}\right)(j=1,2)$ such that $O_{1}^{\prime} \cap O_{2}^{\prime}=\emptyset, P_{1}^{\prime} \cap P_{2}^{\prime}=\emptyset,\left|O_{j}^{\prime}\right|=\left|O_{j}\right|,\left|P_{j}^{\prime}\right|=\left|P_{j}\right|$, and $\left(X^{\prime}, Y^{\prime}\right) \in I_{j}^{\prime} \Leftrightarrow(X, Y) \in I_{j},(j=1,2)$. It is easily seen $\mathcal{B}\left(\mathbb{K}_{j}^{\prime}\right) \cong \mathcal{B}\left(\mathbb{K}_{j}\right)$ and $\mathbb{K}_{j} \cong \mathbb{K}_{j}^{\prime},(j=1,2)$.

Therefore, if both $\mathbb{K}_{1}$ and $\mathbb{K}_{2}$ are geometric, then we obtain the geometric properties of $\mathbb{K}_{1}^{\prime}$ and $\mathbb{K}_{2}^{\prime}$. Let $M_{j}^{\prime}=\left(S_{j}^{\prime}, \mathcal{F}_{j}^{\prime}\right)$ be the matroid produced by $\mathcal{B}\left(\mathbb{K}_{j}^{\prime}\right)$ with the method in [16, p.51, Theorem 1], $(j=1,2)$. We easily obtain $S_{1}^{\prime} \cap S_{2}^{\prime}=\emptyset$ and $N_{j} \cong M_{j}^{\prime},(j=1,2)$. Furthermore, up to the isomorphism, both $M_{1}^{\prime} \leftrightarrow \mathbb{K}_{1}^{\prime}$ and $M_{2}^{\prime} \leftrightarrow \mathbb{K}_{2}^{\prime}$ are correct. Hence $M_{1}^{\prime} \oplus M_{2}^{\prime}$ is true.

Theorem 2.3(2) hints that under the isomorphism, the direct sum of matroids can be considered as the direct sum of contexts.

Corollary 2.4. Let $M$ be a matroid on $S$. Let $M_{j}$ be a matroid on $S_{j}$ where $S_{j} \subseteq S,(j=1,2)$. If $S_{1} \cap S_{2}=\emptyset, S_{1} \cup S_{2}=S$ and $\mathcal{B}\left(\mathbb{K}_{M}\right) \not \mathcal{B}\left(\mathbb{K}_{M_{1}}\right) \times \mathcal{B}\left(\mathbb{K}_{M_{2}}\right)$, then $M$ is connected.

Proof. Otherwise, there are two nontrivial matroids $M_{1}=\left(S_{1}, \mathcal{F}_{1}\right)$ and $M_{2}=$ $\left(S_{2}, \mathcal{F}_{2}\right)$ such that $M=M_{1} \oplus M_{2}, S_{1} \cap S_{2}=\emptyset$ and $S_{1} \cup S_{2}=S$.

In view of Theorem 2.3 , we find $\mathcal{B}\left(\mathbb{K}_{M_{1}}+\mathbb{K}_{M_{2}}\right)=\mathcal{B}\left(\mathbb{K}_{M_{1} \oplus M_{2}}\right)$ up to the isomorphism. Since $\mathcal{B}\left(\mathbb{K}_{M_{1}}+\mathbb{K}_{M_{2}}\right) \cong \mathcal{B}\left(\mathbb{K}_{M_{1}}\right) \times \mathcal{B}\left(\mathbb{K}_{M_{2}}\right)$ holds, we obtain $\mathcal{B}\left(\mathbb{K}_{M}\right)=$ $\mathcal{B}\left(\mathbb{K}_{M_{1} \oplus M_{2}}\right) \cong \mathcal{B}\left(\mathbb{K}_{M_{1}}\right) \times \mathcal{B}\left(\mathbb{K}_{M_{2}}\right)$. This follows a contradiction to the given.

In light of Corollary 2.4, for a matroid $M=(S, \mathcal{F})$ and a submatroid $M_{j}=$ $\left(S_{j}, \mathcal{F}_{j}\right)$ of $M,(j=1,2)$, we receive the Hasse diagrams of $\mathcal{B}(\mathcal{F}, \mathcal{F}, \subseteq), \mathcal{B}\left(\mathcal{F}_{1}, \mathcal{F}_{1}, \subseteq\right)$ and $\mathcal{B}\left(\mathcal{F}_{2}, \mathcal{F}_{2}, \subseteq\right)$ using the algorithms in $[13,15]$. Furthermore, we obtain $\mathcal{B}\left(\mathcal{F}_{1}, \mathcal{F}_{1}, \subseteq\right.$ )$\times \mathcal{B}\left(\mathcal{F}_{2}, \mathcal{F}_{2}, \subseteq\right)$ using the algorithms in [2]. We may easily determine the correction of $\mathcal{B}\left(\mathcal{F}_{M}, \mathcal{F}_{M}, \subseteq\right) \cong \mathcal{B}\left(\mathcal{F}_{1}, \mathcal{F}_{1}, \subseteq\right) \times \mathcal{B}\left(\mathcal{F}_{2}, \mathcal{F}_{2}, \subseteq\right)$ from their Hasse diagrams. Hence, by virtue of Corollary 2.4, we find the connectivity of $M$ and the existence of $M_{1} \oplus M_{2}$.

\section{Concluding Remarks}

With the drawing a concept lattice, i.e the Hasse diagram of a concept lattice, we find under what conditions a construction to be a matroid from different ideas of a matroid such as closed sets, hyperplanes, dual and circuits. In addition, we 
also give an application of drawing concept lattices in the minors, direct sum and connection of matroids. All of these shows that concept lattices can be investigate matroid theory. In the future, we will apply some searching algorithms of concept lattices to reveal the constructions and properties for matroids.

Acknowledgement. This research is Granted by NSF of China (61572011) and NSF of Hebei Province (A2017201007).

\section{REFERENCES}

[1] Faigle, U. and Fujishige, S., "A general model for matroids and the greedy algorithm", Math. Prog., ser. A 119(2)(2009), 353 - 369.

[2] Freese, R., "Automated lattice drawing", http://www.math.hawaii.edu/ ralph

[3] Ganter, B., Stumme, G. and Wille, R., (eds.), Formal Concept Analysis: Foundations and Applications, Springer-Verlag Berlin, Heildelberg, 2005.

[4] Ganter, B. and Wille, R., Formal Concept Analysis: Mathematical Foundations, SpringerVerlag Berlin, Heidelberg, 1999.

[5] Gräzter, G., Lattice Theory, 2nd., Birkhäuser Verlag, Basel, 1998.

[6] Gräzter, G., Lattice Theory: Foundation, Springer Basel AG, Berlin, 2011.

[7] 9th. International Conference on Formal Concept Analysis, Nicosia, Cyprus, May 2-6, 2011.

[8] Lai, H.J., Matroid Theory, Hingher Education Press, Beijing, 2002.(in Chinese)

[9] Mao, H., "An approach to access to a concept lattice via the idea of lattice theory", Univ. Politehnica of Bucharest Sci. Bull., series A, Math. and Phys. 73(2)(2011), 37 - 50.

[10] Mao, H., "Complete atomistic lattices are classification lattices", Algeb. Univ. 68(3-4)(2012), $293-294$

[11] Mao, H., "On closure axioms for a matroid using Galois connections", Math. Commu. $\mathbf{1 4}(2)(2009), 425-432$.

[12] Mao, H., "On lattice-theoretical construction of matroids", Anna. of the Alexandru loan Cuza University-Math. 59(1)(2013), 201 - 208.

[13] Owais, S., Gajdoš, P. and Snášel, V., "Usage of genetic algorithm for lattice drawing", Radim Bělohlávek, Václav Snášel(eds.): CLA 2005, 82-91.

[14] Oxley, J., Matroid Theory, 2nd. ed., Oxford University Press, New York, 2011.

[15] Stumme, G. and Wille, R., "A geometrical heuristic for drawing concept lattices", Lect. Note. in Compu. Sci. 894(1995), 452 - 459.

[16] Welsh, D.J.A., Matroid Theory, Academic Press, London, 1976. 